\title{
Quantification and Quantification Residual biological impurities profile in case of Vaccine, Risk associated impurity toxins, enzymes and oncogenes, should analyse before release vaccine
}

Kailas Narale*

Sr Manager Quality Assurance, Venkateswhwara Hatcheries p.ltd, Pune, India

When implementation by regulators Quantification and Quantification Residual biological impurities profile

WHO biological expert published it Residual biological host cell DNA should not be $10 \mathrm{mg}$ per dose. As per recommendation by WHO Every commercial vaccine batches with compulsory required residual biological impurities especially Host cell DNA and Host Cell Protein have mention result in Certificate of analysis and label claim to understand to common user. Because Impurity are critical harmful to human, Regulator have taken seriously Implementation of analysis of impurities for every commercial batch of vaccine, Practically without analysis vaccine related residual biological impurities especially Host cell DNA and Host cell Protein is release to market.

Host cell DNA Biological residual impurity should not be $10 \mathrm{ng}$ per dose. Practically certificate of analysis vaccine quality control test is not covered, easily analyst can analyses Host cell protein and DNA , impurities but Vaccine specification test is not mentioned regulatory body have implement test for vaccine to avoid risk ,

It is observed in chapter USFDA <1132> impurity profile qualification quantification acceptance limits it is pending to implement in 2017 by regulators, As early as possible vaccine specification to be include -USFDA chapter USFDA <1132>-IN FACT very important test. Host cell DNA Biological residual impurity is MISSING in certificate of analysis of commercial batch of vaccine $[1,2]$.

Any correlation with safety test vaccine and Residual biological impurity of vaccine test?

One cannot correlate two tests for same objective. It is very critical biological impurity host cell DNA and Host Cell Protein will show impact after years may be $2 / 3 / 5$ years. It is risky to release vaccine without knowing Impurity profile, to avoid risk we have analyses biological impurity host cell DNA and Host Cell Protein impurity profile.

\section{Jointly Global biological expert team members of EU/ USFDA/ICH/CDSCO /WHO other regulatory bodies}

Have taken corrective action plan for Implementation It responsibility REGULATOR NOT OF Manufacturers, we can reduce risk associated with biological residual impurity of host cell DNA and Host Cell Protein of vaccine, advance technique are available we have adapt method to test vaccine impurities.
It is Regulatory authorities are responsible to implement by regulation add biological impurity test to be added in specification of vaccine

Residual biological impurity should label claim so common man can understand quality vaccine easily can purchase with help doctor to reduce risk negative impact. Secondary responsibility of manufacturers ethics they should support to regulatory authority to achieve objective product should safe with quality efficacy. To know impurity is right of costumers, request to send regarding corrective action plan long time taken by regulator to implement analysis of residual impurities of commercial batches of vaccine [3].

What is missing vaccine certificate of analysis vaccine regarding impurity profile?

Impurity profile biological Residual Impurity Host cell DNA/ Protein toxins, enzymes and oncogenes. Missed in certificate of analysis.

When implementation about adapt ACCEPTANCE LIMIT for residual biological impurities in vaccine?

1) WHO mentioned Host cell DNA Biological impurity not more than 10 ng per dose

2) Why we are not testing each commercial batch to minimize risk of by testing residual biological impurity in vaccine

3) Qualitative quantitative analysis should have mentioned in certificate of analysis of Vaccine.

4) Regulators known it is very critical but fail to implement USP CHAPTER $<1132>$, failure of regulatory Authority.

5) Certificate of vaccine each lot test parameter to be added in certificate Residual DNA impurity not more than 10 ng per dose.

6) Right consumer to know level of residual biological Host cell DNA impurity as per decided biological WHO USP chapter 1132.

Correspondence to: Kailas Narale, Sr Manager Quality Assurance, Venkateswhwara Hatcheries p.ltd, Pune, India, Tel: +919860363666; E-mail: kvnarale@gmail.com

Received: September 18, 2017; Accepted: October 23, 2017; Published: October 25,2017 
Narale K (2017) Quantification and Quantification Residual biological impurities profile in case of Vaccine, Risk associated impurity toxins, enzymes and oncogenes, should analyse before release vaccine

\section{Is it right of consumer to know the residual biological impurities in vaccine?}

Yes, What about fundament right of customer to know fact about vaccine Finally risk is transfer to end-user who pay money to product, end user paying cost of product risk is not transferrer to manufacturers Regulators Expert.

End-user will suffer by these impurities; all impurities should know to end-user.

Can Global regulatory authority accept challenge to Implement analysis residual biological impurity with acceptance limit?

When USP chapter 1132 implement to identify qualitative quantitative analysis Host cell DNA/and Protein we have analytical tool procedure are available, why regulatory bodies delay to implement when it will be implement for safety of end-user [4,5].

\section{Summary}

Residual impurity profile should analyses; use should know other viruses, foreign proteins, residual segments of DNA and RNA, toxins, enzymes and oncogenes. It has proved impossible to remove these contaminants. This is well known to the regulatory authorities, greatly concerns many of their experts, but these very serious concerns are not communicated to the user.

\section{References}

1. USP guidelines (2007) United states pharmacopeia. Chapter 4, Astra Edu Library.

2. WHO (2017) Biologicals, biotherapeutics. World health organization.

3. Medical Veritas 5 (2008) The Dangerous Impurities of Vaccines - Medical Veritas.

4. GEN (2013) Testing for Residual Host Cell Protein \& DNA. Genetic engineering and biotechnology.

5. USP (2017) Residual Host Cell Protein Measurement in Biopharmaceuticals.

Copyright: $\odot 2017$ Narale K. This is an open-access article distributed under the terms of the Creative Commons Attribution License, which permits unrestricted use, distribution, and reproduction in any medium, provided the original author and source are credited. 